\title{
Plasma metanephrines: a novel and cost- effective test for pheochromocytoma
}

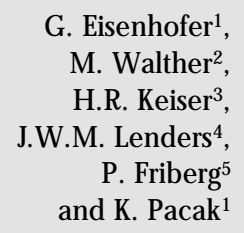

\author{
${ }^{1}$ Clinical Neurocardiology Section, $\mathrm{N}$ ational Institute of Neurological Disorders and \\ Stroke, $2 \mathrm{U}$ rologic $\mathrm{O}$ ncology Branch, N ational Cancer Institute, and \\ ${ }^{3} \mathrm{H}$ ypertension Endocrine Branch, National Heart, Lung and Blood Institute, \\ National Institutes of Health, Bethesda, MD, USA \\ ${ }^{4}$ Department of General Internal Medicine, St. Radboud U niversity H ospital, \\ Nijmegen, The Netherlands \\ ${ }^{5}$ Department of Clinical Physiology, University of Göteborg, Göteborg, Sweden
}

\section{Correspondence}

G. Eisenhofer

Building 10, Room 6N 252

National Institutes of $\mathrm{H}$ ealth

10 Center DR, MSC-1620

Bethesda, MD 20892-1620

USA

Fax: +1-301-402-0180

E-mail: graeme@ catecholamine.org

Presented at the First

International M eeting on Adrenal

Disease: Basic and Clinical

Aspects, Ribeirão Preto, SP, Brazil,

August 31-September 2, 1999.

Received December 20, 1999

Accepted April 4, 2000

\section{Abstract}

Pheochromocytomas are rare chromaffin cell tumors that nevertheless must be excluded in large numbers of patients who develop sustained or episodic hypertension as well as in many others with suggestive symptoms or with a familial history of pheochromocytoma. Diagnosis of pheochromocytoma depends importantly on biochemical evidence of excess catecholamine production by a tumor. Imperfect sensitivity and specificity of commonly available biochemical tests and the low incidence of the tumor among the tested population mean that considerable time and effort can be expended in confirming or ruling out pheochromocytoma in patients where the tumor is suspected. Measurements of plasma free metanephrines provide a superior test compared to other available tests for diagnosis of pheochromocytoma. In particular, the high sensitivity of plasma free metanephrines means that a normal test result reliably excludes all but the smallest of pheochromocytomas so that no other tests are necessary. Measurements of plasma free metanephrines, when systematically combined with other diagnostic procedures outlined in this review, provide a more efficient, reliable and cost-effective approach for diagnosis of pheochromocytoma than offered by previously available approaches.

\section{Key words}

- Pheochromocytoma

- Hypertension

- Sympathetic nervous system

- Adrenal gland

- Metanephrines

- Catecholamines

\section{Introduction}

Pheochromocytomas are chromaffin cell tumors typically arising within the adrenal medulla. These tumors are a rare cause of hypertension that nevertheless must be excluded in a significant proportion of the $20 \%$ of the adult population of western countries who develop high blood pressure. In the United States alone this amounts to about 800,000 cases of newly diagnosed hypertension each year in which pheochromocytoma may represent a correctable cause of high blood pressure. In reality, however, it is not feasible or cost effective to screen for pheochromocytoma in every patient with hypertension, particularly when commonly available tests do not always detect the tumor. Therefore, this diagnosis is most often only considered when a patient shows episodic hypertension, fails to respond to antihypertensive therapy, has a hypertensive episode during anesthesia or surgery or when there are other suggestive symptoms, such as head- 
ache, sweatiness, anxiety, palpitations or tachycardia. It also must be considered that some patients, particularly those with a familial predisposition to pheochromocytoma, may not show increased blood pressure or the typical symptoms of a pheochromocytoma. In these patients biochemical diagnosis of the tumor can be particularly troublesome.

Diagnosis of pheochromocytoma typically requires confirmation by several tests, perhaps the most important being biochemical evidence of excessive catecholamine production by the tumor. This is usually achieved from measurements of catecholamines and certain catecholamine metabolites in urine or plasma (Table 1). However, the catecholamines, norepinephrine and epinephrine, are also produced by sympathetic nerves and the adrenal medulla and are thus not specific to pheochromocytomas. Therefore, high levels of catecholamines and their metabolites may be produced by a variety of conditions or disease states involving increased release of catecholamines from sympathetic nerves or the adrenal medulla (1). Sometimes pheochromocytomas may be "silent", that is they may not produce catecholamines in amounts sufficient to produce a positive biochemical test result or the associated typical clinical signs and symptoms. Also, many pheochromocytomas secrete catecholamines episodically; between episodes, plasma concentrations or urinary excretion of catecholamines may be normal. Thus, tests of plasma or urinary catecholamines and urinary metabolites of catecholamines do not always reliably exclude or confirm the presence of a tumor (2-7). A more recently developed biochemical test involving measurements of

Table 1 - Biochemical tests for diagnosis of pheochromocytoma.

Reference ranges indicate lower and upper reference limits of a normal population commonly estimated from the $95 \%$ confidence intervals. Reference ranges vary among laboratories. VMA, Vanillylmandelic acid.

\begin{tabular}{|c|c|c|}
\hline Biochemical test & Measurement method & Reference range \\
\hline Urine catecholamines & HPLC & \\
\hline Norepinephrine & & 15-80 mg/day \\
\hline Epinephrine & & $0-20 \mu \mathrm{g} / \mathrm{day}$ \\
\hline Urine fractionated metanephrines & HPLC & \\
\hline Normetanephrine-sulfate & & $44-540 \mu \mathrm{g} / \mathrm{day}$ \\
\hline $\begin{array}{l}\text { Metanephrine-sulfate } \\
\text { (Sum of free plus sulfate-conjugated } \\
\text { metanephrine or normetanephrine) }\end{array}$ & & $26-230 \mu \mathrm{g} / \mathrm{day}$ \\
\hline $\begin{array}{l}\text { Urine total metanephrines } \\
\text { (Combined sums of free plus sulfate-conjugated } \\
\text { metanephrine and normetanephrine) }\end{array}$ & Spectrofluorimetry & $0-1.2 \mathrm{mg} / \mathrm{day}$ \\
\hline Urine VMA & Spectrofluorimetry & 0-7.9 mg/day \\
\hline Plasma catecholamines & HPLC & \\
\hline Norepinephrine & & $80-498 \mathrm{pg} / \mathrm{ml}$ \\
\hline Epinephrine & & $4-83 \mathrm{pg} / \mathrm{ml}$ \\
\hline Plasma free metanephrines & HPLC & \\
\hline Normetanephrine & & $18-112 \mathrm{pg} / \mathrm{ml}$ \\
\hline Metanephrine & & $12-61 \mathrm{pg} / \mathrm{ml}$ \\
\hline Plasma deconjugated metanephrines & HPLC & \\
\hline Normetanephrine-sulfate & & $610-3170 \mathrm{pg} / \mathrm{ml}$ \\
\hline Metanephrine-sulfate & & $316-1706 \mathrm{pg} / \mathrm{ml}$ \\
\hline
\end{tabular}


plasma free normetanephrine and metanephrine, the respective metabolites of norepinephrine and epinephrine, offers advantages over other tests for diagnosis of pheochromocytoma $(5,7)$. As reviewed here, the test may offer a cost-effective approach for more effective screening for the tumor than provided by other tests.

\section{Disposition of catecholamines derived from the sympatho-adrenal system and pheochromocytomas}

Understanding the utility and limitations of biochemical tests for diagnosis of pheochromocytoma can benefit from an understanding of catecholamine release and metabolism under normal conditions as well as in disease states associated with elevated catecholamine release. Both norepinephrine and epinephrine are metabolized by a multiplicity of pathways catalyzed by an array of enzymes, resulting in a considerable number of different metabolites, only some of which are routinely used for diagnosis of pheochromocytoma (Figure 1).

Most important to an understanding of the utility of catecholamines and their metabolites in the diagnosis of pheochromocytoma is an appreciation of how catecholamines are metabolized differently within nerves and other cells, before and after their entry into the bloodstream and among various organs and tissues, including chromaffin cells and pheochromocytoma tumor cells. Pheochromocytomas differ from sympathetic nerves or central nervous system noradrenergic nerves but are similar to adrenomedullary cells in that they secrete catecholamines directly into the bloodstream. In contrast, the norepinephrine formed or released from sympathetic nerves acts and is metabolized locally so that only a small proportion escapes local removal and metabolism to diffuse into the bloodstream (8-10) (Figure 2).

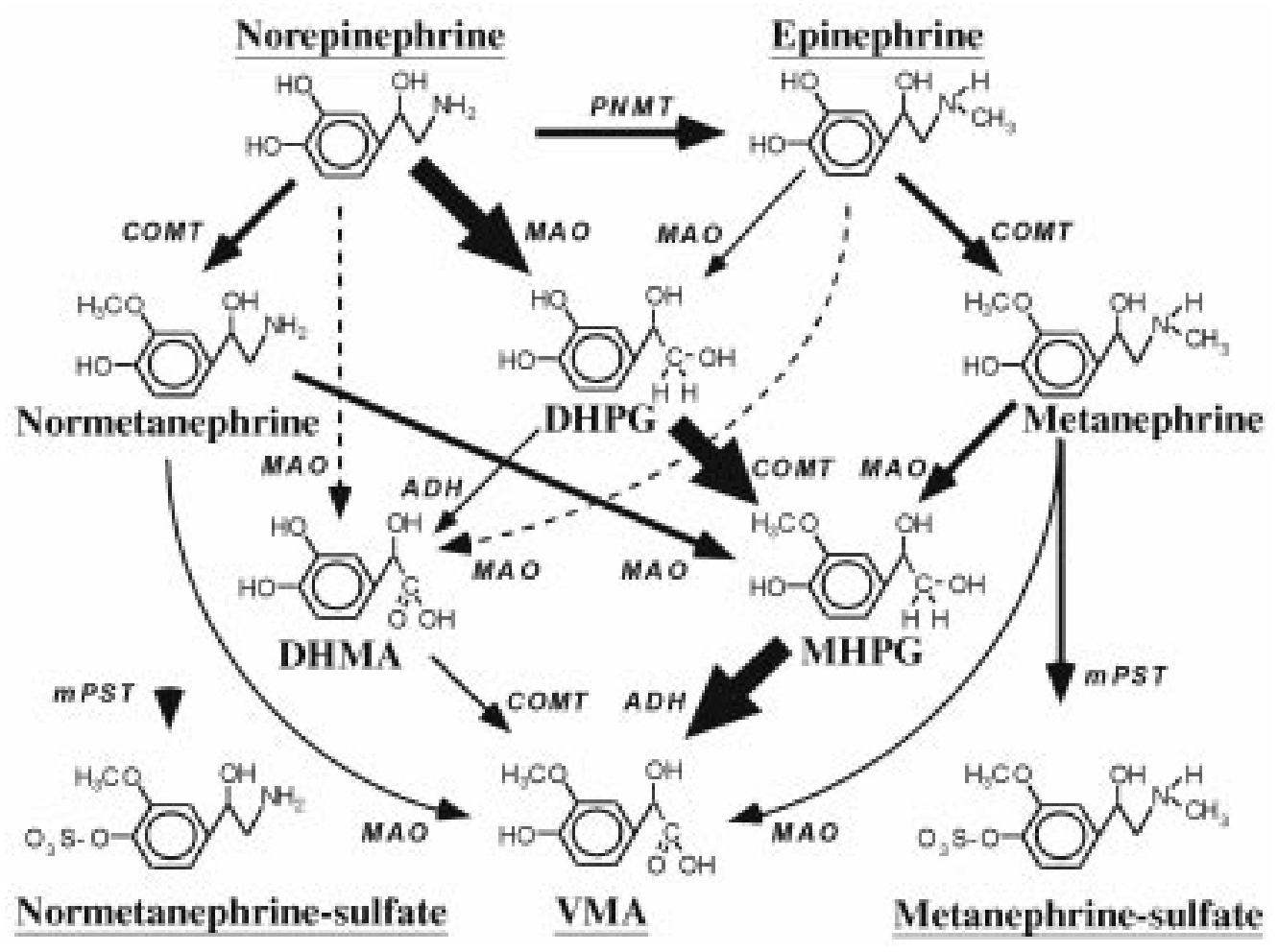

Figure 1 - Pathways of metabolism of norepinephrine and epinephrine. Enzymes responsible for each pathway are indicated by the arrowheads. The more solid arrows indicate the more major pathways of metabolism while the dotted arrows indicate pathways of negligible importance. Compounds that are routinely measured in urine or plasma for diagnosis of pheochromocytoma are underlined. All compounds except VMA are sulfate conjugated but only pathways of sulfate conjugation for normetanephrine and metanephrine are shown. PNMT, Phenolethanolamine-N-methyltransferase; MAO, monoamine oxidase; COMT, catechol-O-methyltransferase; ADH, alcohol dehydrogenase; $\mathrm{m}-\mathrm{PST}$, monoamine preferring phenolsulfotransferase; DHPG, 3,4-dihydroxyphenylglycol; DHMA, 3,4-dihydroxymandelic acid; MHPG, 3-methoxy-4-hydroxyphenylglycol; VMA, vanillylmandelic acid. 
Since norepinephrine is the predominant catecholamine secreted by pheochromocytomas, an understanding of its metabolism after release and production within sympathetic nerves, as compared with after release directly into the bloodstream by a pheochromocytoma, is particularly important. Comparison of removal and metabolism of catecholamines by neuronal and extraneuronal cells has indicated that the sympathetic nerves are far more important than the non-neu- ronal cells for inactivation of neuronally released norepinephrine $(9,10)$. This means that most of the norepinephrine produced and released by nerves is metabolized within the nerves themselves (Figure 2). Because monoamine oxidase (MAO) is the only catecholamine-metabolizing enzyme present in sympathetic nerves, the norepinephrine metabolized within these nerves is all converted to dihydroxyphenylglycol (DHPG) (11-13). As a consequence, the DHPG appearing in

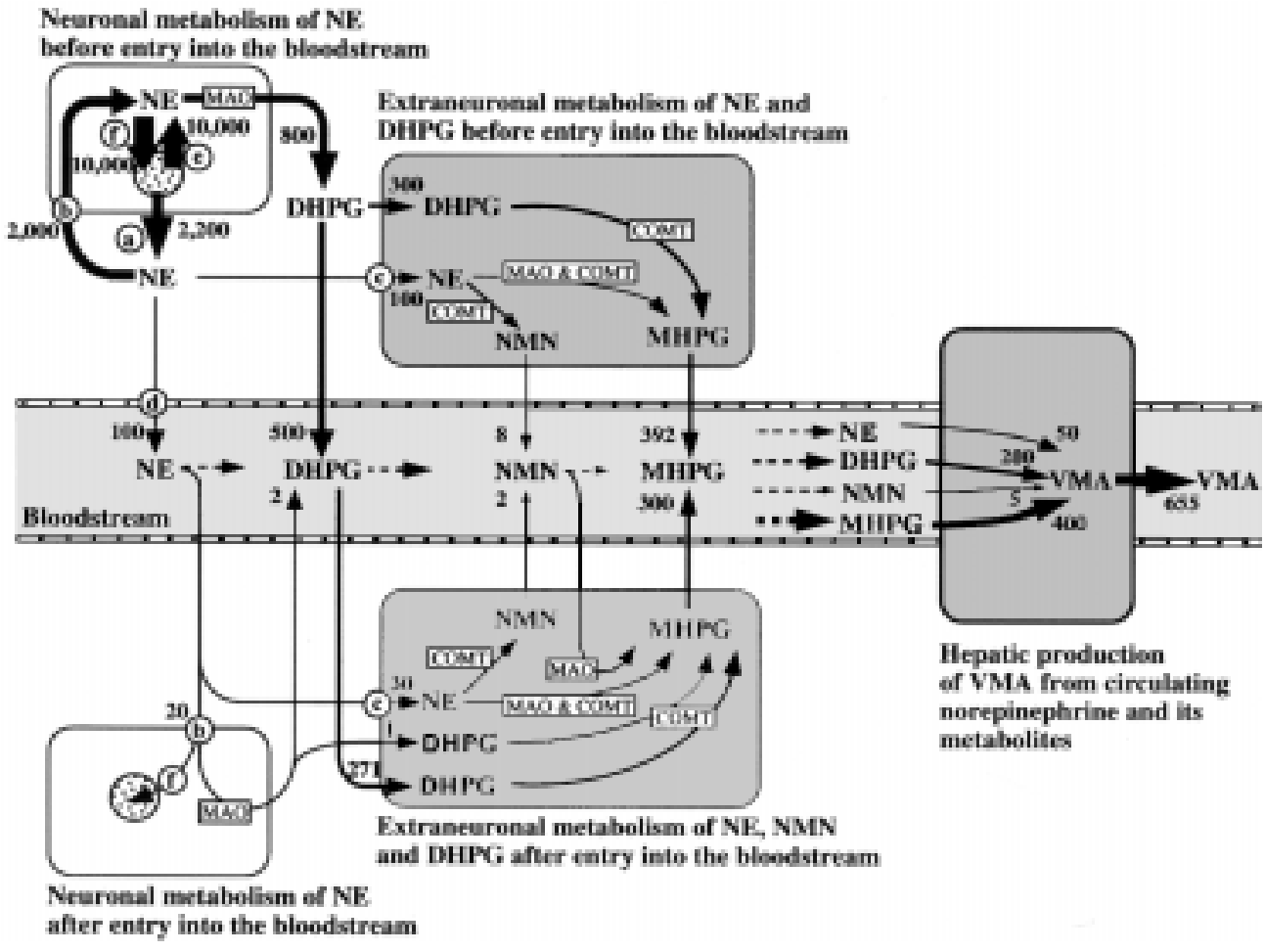

Figure 2 - Quantitative diagram showing neuronal and extraneuronal pathways of norepinephrine (NE) metabolism before and after entry into the bloodstream. Numbers indicated by arrowheads show relative rates of each process as derived from previously published data. The diagram does not show sulfate conjugation pathways of metabolism that are particularly important for normetanephrine (NMN) and 3-methoxy-4-hydroxyphenylglycol (MHPG). The diagram also does not include the contribution of the adrenals to circulating NE (9\%) and free NMN (24-40\%). Most of the NE released by sympathetic nerves (a) is removed by neuronal uptake (b) and a much smaller amount is removed by extraneuronal uptake (c) so that only a small portion escapes to enter the bloodstream (d). Most of the NE recaptured by sympathetic nerves is sequestered into storage vesicles by the vesicular monoamine oxidase (MAO) transporter (f) and a smaller proportion is metabolized intraneuronally to 3,4-dihydroxyphenylglycol (DHPG). However, considerably more of the NE that is sequestered into storage vesicles or metabolized intraneuronally to DHPG is derived from transmitter leaking from storage vesicles (e) than from reuptake (b). Very little circulating DHPG is derived from metabolism of circulating NE, whereas a significant proportion of the small amounts of circulating free NMN is formed from circulating NE. MHPG is mainly derived from O-methylation of DHPG before and after its entry into the bloodstream. Vanillylmandelic acid (VMA) is mainly derived from metabolism of MHPG and DHPG in the liver. COMT, Catechol-O-methyltransferase. 
plasma is almost exclusively produced in sympathetic nerves, whereas the additional presence of catechol-O-methyltransferase in extraneuronal cells means that normetanephrine is exclusively produced from norepinephrine in extraneuronal cells, such as smooth muscle cells or liver cells $(9,11)$. Much of the DHPG formed in nerves is metabolized further to 3-methoxy-4-hydroxyphenylglycol (MHPG) by catechol-O-methyltransferase in extraneuronal cells (14).

The above considerations combined with the series nature of neuronal and extraneuronal removal and metabolism (15) explain why very little of the DHPG in plasma $(<1 \%)$ is derived from neuronal metabolism of norepinephrine released directly into the bloodstream (Figure 2). Thus, release of norepinephrine from a pheochromocytoma directly into the bloodstream causes only small increases in DHPG compared with release of norepinephrine from nerves (16). Hence, patients with pheochromocytoma and high norepinephrine levels often have normal or only slightly elevated plasma concentrations of DHPG $(16,17)$. Findings of a high norepinephrine combined with a normal DHPG therefore provide supportive evidence that an increased plasma concentration of norepinephrine is not due to excessive release from sympathetic nerves and might rather reflect a tumor $(16,18,19)$.

Vanillylmandelic acid (VMA), the major end-product of norepinephrine and epinephrine metabolism, is produced almost exclusively from the removal and metabolism by the liver of catecholamines and their metabolites that circulate in the bloodstream (20) (Figure 2). This is because the enzyme responsible for formation of VMA from MHPG, alcohol dehydrogenase, is localized to the liver $(21,22)$. The substantial production of VMA from circulating DHPG and MHPG, most of which is derived from neuronal norepinephrine metabolism, explains why VMA is a relatively insensitive marker for pheochromocytoma compared with the precursors norepinephrine, epinephrine, normetanephrine and metanephrine (23-27).

Normally at least $90 \%$ of metanephrine and up to $40 \%$ of normetanephrine are formed from metabolism of epinephrine and norepinephrine within the adrenals before release of these catecholamines into the circulation $(28,29)$. This makes the adrenal medulla the single largest source of both normetanephrine and metanephrine in the body, exceeding the contribution of the liver (28). Both adrenomedullary and pheochromocytoma tumor cells contain high quantities of catecholO-methyltransferase (30), the enzyme that is responsible for O-methylation of catecholamines to form normetanephrine and metanephrine (31). The catechol-O-methyltransferase is localized within chromaffin cells so that the normetanephrine and metanephrine from these sources are derived from catecholamines leaking from stores into the chromaffin cell cytoplasm.

In patients with pheochromocytoma, over $94 \%$ of the elevated plasma concentrations of normetanephrine or metanephrine are derived from metabolism of catecholamines by the catechol-O-methyltransferase within pheochromocytoma tumor cells and not by actions of extra-adrenal catechol-O-methyltransferase on catecholamines released by tumors into the circulation (30). This means that production of normetanephrine and metanephrine is an ongoing process within pheochromocytoma tumor cells, independent of catecholamine release. This explains why plasma concentrations of normetanephrine and metanephrine are relatively insensitive markers of increased norepinephrine release by nerves or increased epinephrine release from the adrenals or of a paroxysmal attack associated with large increases in catecholamine release from a pheochromocytoma (30). However, this also means that even when tumors are not secreting catecholamines into the bloodstream they are nevertheless constantly metabolizing catecholamines to normetanephrine and/or metanephrine. 


\section{Sensitivity of biochemical tests}

The sensitivity of a test for diagnosis of pheochromocytoma is indicated by the proportion of patients with a tumor who have a positive test result (i.e., an elevated plasma concentration or urinary output). A sensitivity of $100 \%$ indicates that in all cases of patients who have a tumor the test will be positive. A sensitivity of $100 \%$ also means that a normal test result reliably excludes the presence of a tumor. Importantly, it must be realized that a high sensitivity of $100 \%$ does not necessarily prove the existence of a pheochromocytoma in all patients who have a positive test result. This depends on the specificity of the test.

A problem with use of plasma or urinary catecholamines for diagnosis of pheochromocytoma is that some tumors are quiescent and may not secrete large amounts of catecholamines while other tumors secrete catecholamines episodically. Thus, plasma lev-

Figure 3 - Plasma concentrations of normetanephrine, metanephrine, norepinephrine and epinephrine in 29 patients with von Hippel-Lindau (VHL) disease and pheochromocytoma (grey bars) and 10 patients with multiple endocrine neoplasia type 2 (MEN-2) and pheochromocytoma (black bars). Each bar represents a concentration of one of the 4 analytes for a single patient. The lower dashed lines show the upper reference limits (URL) of normal levels. Concentrations above these lines are high and those below are normal. The upper dashed lines show levels at 5 times the upper reference limits. Concentrations above the horizontal lines almost certainly indicate the presence of a pheochromocytoma.

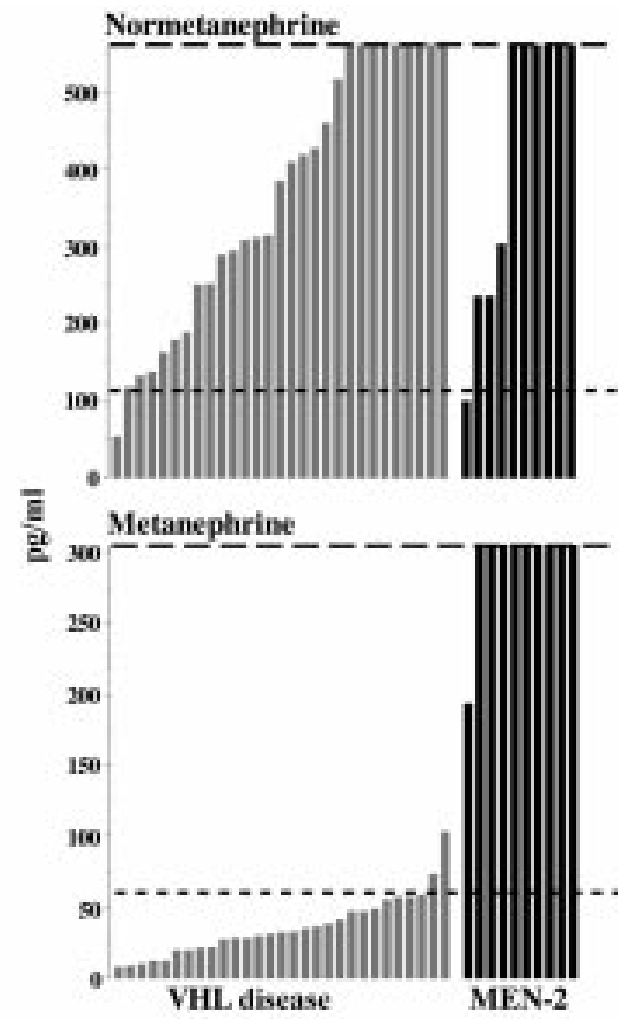

els and urinary outputs of catecholamines are normal in some patients with pheochromocytoma and the presence of a pheochromocytoma cannot be reliably excluded using measurements of plasma or urinary catecholamine concentrations (2-7,23-27). In contrast, the metanephrines (either normetanephrine or metanephrine or both) are constantly produced by the actions of catechol-O-methyltransferase on catecholamines leaking from stores within tumor cells and therefore show much more consistent increases above normal in patients with pheochromocytoma than plasma catecholamines (5,7) (Figure 3). This means that measurements of plasma normetanephrine and metanephrine reliably exclude the presence of all but the smallest of pheochromocytomas. Where excluded, no other tests are necessary. Thus, measurements of plasma metanephrines avoid a missed diagnosis and minimize the need to run multiple diagnostic tests to exclude the presence of a tumor. This also means that a

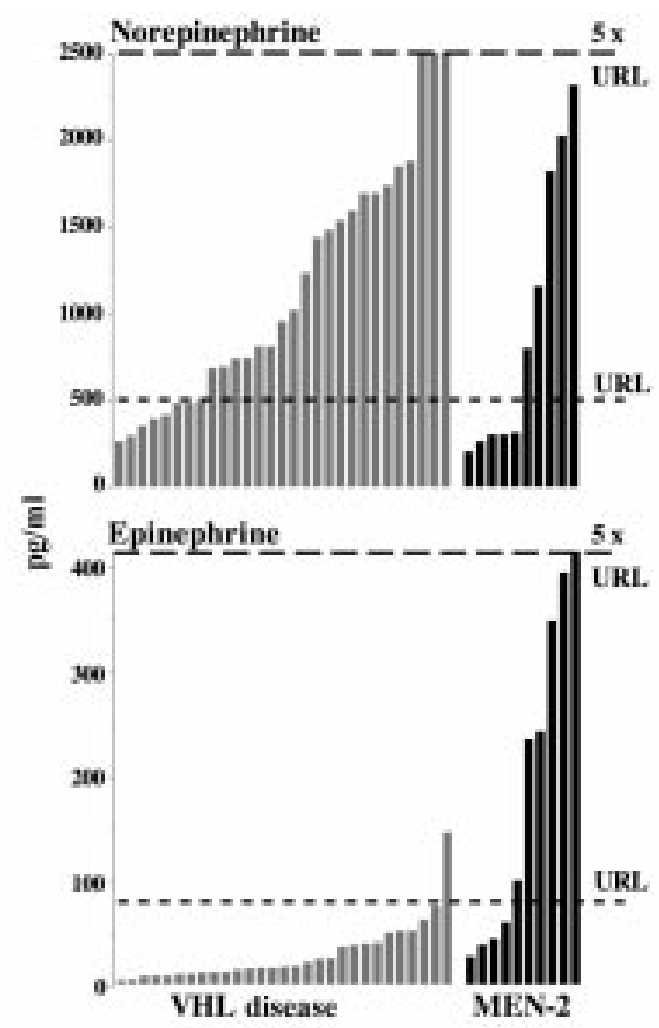


person with normal plasma concentrations of normetanephrine and metanephrine can be fairly confident of not having a pheochromocytoma.

Since the free metanephrines are formed extraneuronally, and to a large extent within chromaffin tissues (e.g., adrenal medulla and pheochromocytomas), these metabolites are also more sensitive markers for a pheochromocytoma than the other catecholamine metabolites that are derived mainly from neuronal sources.

Urinary metanephrines are commonly measured after acid hydrolysis and thus largely represent sulfate-conjugated normetanephrine and metanephrine. A substantial amount of the normetanephrine-sulfate is derived from sulfate conjugation of normetanephrine produced in parts of the body other than the adrenal medulla or pheochromocytoma tumor chromaffin tissue. Therefore, the sulfate-conjugated normetanephrine, as commonly measured in urine, is a less sensitive marker of pheochromocytoma than the free normetanephrine measured in plasma. In particular, measurements of the combined sum of urinary outputs of normetanephrine and metanephrine in sulfate-conjugated plus free form (commonly known as urinary total metanephrines), as measured by outdated spectrofluorometric methods, are not sensitive tests of a pheochromocytoma; therefore, they have limited value in the initial work-up of a patient suspected of having a pheochromocytoma. The low sensitivity of urinary VMA also makes this test less than satisfactory for the initial biochemical diagnosis of a pheochromocytoma.

\section{Specificity of biochemical tests}

As distinct from sensitivity, the specificity of a biochemical test indicates how likely it is that a patient with a positive test result has a pheochromocytoma. Thus, a high specificity of $100 \%$ would indicate that the test reliably proves the presence of a pheochro- mocytoma in all patients with a positive test result. Importantly, it must be realized that a high specificity of $100 \%$ does not necessarily mean that all patients with a pheochromocytoma will have a positive test result. This is a matter of sensitivity.

Since upper reference limits of normal of most diagnostic tests are typically established from the $95 \%$ confidence intervals (with $2.5 \%$ below and $2.5 \%$ above) of a range of values determined in a reference population (see Table 1 for normal reference limits), it can be expected that all tests will give at least a $2.5 \%$ incidence of false-positive results (i.e., $2.5 \%$ of all test results will show an elevated value that might suggest the presence of a tumor when none is really present). This means that no biochemical test can be expected to have $100 \%$ specificity and the best that might be expected would be $97.5 \%$. In practice, however, because many of the patients screened for pheochromocytoma are ill and may have activated sympatho-adrenomedullary systems, the specificity of tests of catecholamines and metabolites is often lower than $97.5 \%$.

Since the catecholamines and their metabolites are normally produced by sympathetic nerves and the adrenal medulla, none of these compounds are highly specific for the presence of a pheochromocytoma. In particular, plasma and urinary catecholamines may be elevated by a variety of physiological, pharmacological and pathological conditions (1). Nevertheless, the combination of measurements of catecholamines and of certain metabolites can be useful in helping to distinguish elevated catecholamines due to a tumor from elevated catecholamines secondary to increased release from sympathetic nerves or the adrenal medulla.

Increased release of catecholamines from sympathetic nerves or the adrenal gland secondary to exercise, mental stress, low blood pressure, low blood volume, low blood glucose or certain drugs represent conditions 
that may elevate plasma and urinary catecholamines and cause false-positive test results. During blood sampling and 24-h urine collections these influences must be avoided. Upright posture is another important determinant of catecholamine release, increasing plasma norepinephrine by as much as 3 -fold above values in the lying position. Therefore, collection of blood samples should be performed with the patient resting quietly in the lying position for at least $20 \mathrm{~min}$ before sampling with an indwelling intravenous catheter previously inserted to avoid any possible acute stress associated with insertion of the needle.

A wide variety of pathological conditions may be associated with elevated plasma and urinary catecholamines. Congestive heart failure, renovascular hypertension, hypernoradrenergic hypertension, shock, sepsis, dumping syndrome, sleep apnea, anxiety neurosis and panic disorder are some of the syndromes that may be associated with elevated plasma or urinary catecholamines and clinical symptoms suggestive of a pheochromocytoma.

The above physiological and pathological conditions can also lead to elevated production of metanephrines and VMA, with attendant false-positive test results for these metabolites. However, because production of these metabolites is somewhat independent of catecholamine release from the sympathetic nerves or the adrenal medulla, their proportional increases are typically less than those in catecholamines. The metanephrines, in particular, are relatively poor markers of increased release of catecholamines from sympathetic nerves or the adrenal gland $(29,30)$. Theoretically, this should make the metanephrines less prone to false-positive results in physiological and pathological states associated with sympatho-adrenomedullary activation. However, there are other factors to consider that may additionally contribute to false-positive results for these tests. Deficiency or pharmacological inhibition of MAO increases both urinary deconjugated and plasma free metanephrines due to impaired breakdown of the metabolite by MAO and increased shunting of metabolism through O-methylation pathways $(32,33)$. Severe renal failure can be particularly problematic in patients suspected of having a pheochromocytoma, making urine test results unreliable (34) or urine collection impossible. In these patients biochemical diagnosis typically requires assays of plasma catecholamines and metanephrines. However, the sympathetic nerves can be activated in these patients, resulting in elevated plasma norepinephrine concentrations. Also, end-products of catecholamine metabolism, that depend on elimination by the kidneys such as the sulfate-conjugated metanephrines - tend to build up in plasma to very high levels (35).

During interpretation of biochemical test results it is important to keep in mind that these are numerical values and should not be considered as simply either negative (within the normal range) or positive (above the normal range). Rather, the magnitude of an increase above normal values should also be considered. For example, a patient presenting with suggestive symptoms and a plasma norepinephrine concentration of over 2500 $\mathrm{pg} / \mathrm{ml}(14.8 \mathrm{pmol} / \mathrm{ml})$, approximately 5 times above the upper reference limit of normal, is far more likely to have a pheochromocytoma than a patient with the same symptoms and a plasma norepinephrine concentration just above the upper reference limits. The few conditions where plasma norepinephrine can reach such high levels (e.g., hypernoradrenergic hypertension, end-stage congestive heart failure, circulatory shock) are easily excluded. In patients with pheochromocytoma, plasma concentrations of normetanephrine and metanephrine typically show much larger relative increases above the upper reference limits than observed for tests of catecholamines, urinary metanephrines and VMA. This indicates that at the higher limits more specific for a tumor, measurements of 
plasma normetanephrine and metanephrine provide better proof (i.e., are more specific) of a pheochromocytoma than other available tests.

\section{Diagnostic procedural recommendations}

\section{Initial biochemical testing}

With issues of sensitivity and specificity in mind, as well as consideration of the potential dangers of a pheochromocytoma and the rarity of the tumor, the most important consideration in the choice of an initial biochemical test is the reliability of the test for exclusion of pheochromocytoma. In pheochromocytoma, a missed diagnosis due to a false-negative test result can have catastrophic consequences for the patient. In contrast, a single false-positive test result can be refuted by further tests. Therefore, a suitably sensitive biochemical test remains the first choice in the initial work-up of the patient suspected to be harboring a pheochromocytoma. With most available biochemical tests, however, confirming the absence of a tumor can be more problematic than confirming the presence of a tumor.

Because of the high sensitivity of the test, our recommendation is to use HPLC measurements of plasma free normetanephrine and metanephrine as the initial biochemical test of choice. This test can be combined with or followed by HPLC measurements of plasma catecholamines collected under appropriately controlled circumstances for further interpretation of any elevations in plasma free metanephrines. To circumvent falsepositive test results, appropriate consideration should always be given to interference with assay results by any drugs that the patient may be taking.

If plasma free metanephrines have been run, and they are well within the normal range, then it is highly unlikely that the patient has anything but a very small tumor (most likely less than $1 \mathrm{~cm}$ in diameter) and there should be little need to run further tests at this stage. On the other hand, normal results for each of the other biochemical tests, even when performed in combination, are still possible in some patients with a pheochromocytoma. Thus, if plasma free metanephrines have not been run, but the other above test results are all normal, then it is still possible that the patient has a pheochromocytoma.

\section{Secondary diagnostic tests}

If tests of plasma free metanephrines or any of the above combinations of test results are positive then further tests must be considered to exclude or prove the existence of a pheochromocytoma. At this stage it is again important to consider any potential associated clinical conditions or influences of medications that may cause a false-positive test result. Additional considerations include the magnitude of increase above normal of biochemical test results (Figure 3) along with the pattern of alterations in biochemical parameters which may be used together to make some kind of qualitative assessment of the likelihood that the patient has a tumor. From this a strategy for further testing can be developed, including imaging studies to localize the tumor, provocation tests and further biochemical tests. Ultimately, it is a combination of biochemical and radiological tests combined with clinical assessment of symptoms, signs and associated conditions that provides the clinician with sufficient evidence to establish or refute the diagnosis of pheochromocytoma.

\section{Ruling out sympatho-adrenomedullary activation}

Use of the clonidine suppression test is particularly useful when there is suspicion that elevated plasma concentrations of norepinephrine are secondary to increased re- 
lease of the transmitter from sympathetic nerves rather than from a pheochromocytoma. The basis of the test is simple and involves oral administration of the drug, clonidine, with measurements of plasma norepinephrine concentrations before and $3 \mathrm{~h}$ after the drug. Clonidine acts on centers in the brain to decrease the release of norepinephrine from sympathetic nerve terminals. The drug does not decrease release from a pheochromocytoma. Thus, the drug will decrease elevated plasma norepinephrine concentrations in a patient whose elevations are due to increased release of the transmitter from sympathetic nerves; a pheochromocytoma may then be excluded. In a patient with high plasma norepinephrine concentrations due to release from a pheochromocytoma, the norepinephrine concentrations are little affected by clonidine.

Other indicators of sympatho-adrenomedullary activation, rather than a pheochromocytoma, are the distinct patterns of biochemical test results that accompany sympathetic activation as opposed to a tumor. Patterns of biochemical test results that are more suggestive of increased release from sympathetic nerves or the adrenal gland than from a tumor (such as occurs in hypernoradrenergic hypertension, renovascular hypertension, congestive heart failure, panic disorder, dumping syndrome, and other conditions) include proportionally larger elevations of plasma norepinephrine or epinephrine than of plasma normetanephrine or metanephrine. In sympathetic activation, increases in plasma DHPG that parallel the increases in norepinephrine typically reflect a source of the elevated norepinephrine from sympathetic nerves rather than from a pheochromocytoma. This pattern of biochemical test results combined with a negative clonidine suppression test result (i.e., a substantial decrease in plasma norepinephrine after clonidine) is highly suggestive of sympathetic activation rather than a tumor, and, unless imaging studies suggest otherwise, a pheochromocytoma can be excluded and no further tests should be necessary.

\section{Locating the tumor}

If plasma or urine levels of catecholamines and metanephrines are high, suppression tests and/or stimulation tests are positive (i.e., plasma norepinephrine does not decrease after clonidine, plasma norepinephrine shows a substantial increase after glucagon), or if the biochemical pattern of test results is indicative of a tumor rather than increased release from sympathetic nerves or the adrenal gland, then it is important to localize the tumor for subsequent surgical removal.

In most cases of positive initial biochemical test results, where clinical suspicion of a pheochromocytoma remains reasonable, a CT or magnetic resonance imaging (MRI) scan of the entire abdomen may be immediately appropriate. A finding of an adrenal or abdominal mass together with suggestive clinical signs and symptoms and highly elevated catecholamines and their metabolites and an appropriate pattern of changes in metabolites and catecholamine precursors (i.e., larger relative increases in plasma free metanephrines than catecholamines and/or normal plasma DHPG levels) might be all that is needed at this stage to justify surgery. However, in many cases it is also appropriate to follow up with MIBG scintigraphy preferably using the 123-iodine-labeled compound rather than the 131-iodine-labeled compound - to establish more reliably that a located mass is a pheochromocytoma or to locate an extra-adrenal tumor or metastases.

\section{Identifying the "silent" pheochromocytoma}

In occasional cases of sporadic pheochromocytoma and in some incidentalomas, but quite frequently during routine screening of patients with von Hippel-Lindau (VHL) disease or multiple endocrine neoplasia type 2 (MEN-2), a small adrenal mass may be 
present without any of the symptoms and signs associated with a pheochromocytoma $(7,36,37)$. In these patients, the pheochromocytoma is "silent" and may not produce catecholamines in amounts sufficient to cause typical symptoms or a positive test result for plasma or urinary catecholamines, urinary VMA or urinary metanephrines. These patients, however, typically show some elevation of either plasma free normetanephrine (VHL patients) or both plasma free normetanephrine and metanephrine or only metanephrine (MEN-2 patients). Should this not be sufficient reason to operate, then it is important to follow these patients carefully over time, since as the tumor(s) enlarge plasma free normetanephrine and/or metanephrine increases, providing a stronger reason to operate. Use of glucagon-stimulation tests and other imaging techniques with higher specificity than $\mathrm{CT}$, such as MIBG scintigraphy, may also be useful to identify a "silent" pheochromocytoma. However, the likelihood of false-negative results for the glucagon-stimulation test and MIBG scanning, particularly in cases of small tumors, points to the need for careful follow-up studies in patients suspected of harboring a "silent" pheochromocytoma.

\section{Summary: the cost-effectiveness of plasma metanephrines}

In the United States only about $10 \%$ and perhaps as many as $20 \%$ of all newly diagnosed hypertensives are screened for pheochromocytoma. Nevertheless, this represents about 80,000 to 160,000 patients each year who are tested. Testing is also often carried out in normotensive individuals who complain of other symptoms suggestive of a pheochromocytoma (e.g., panic attacks). Patients with familial conditions that predispose to pheochromocytoma, such as multiple endocrine neoplasia type 2 and von Hippel-Lindau disease, represent another group in whom testing for the tumor is called for on an annual basis. Thus, currently in the United States alone somewhere between 100,000 and 200,000 individuals are screened for the tumor every year. Of these only between 800 to 1600 patients are subsequently found to have the tumor, representing an incidence of the tumor in the tested population of about 1 in 1000 cases. Because of the limitations of commonly available tests many other patients with pheochromocytoma are never diagnosed and die prematurely as a consequence of the clinical complications of the tumor.

The cost-effective potential of plasma metanephrines as the initial screening test for detection of pheochromocytoma primarily depends on the high sensitivity of the test. This high sensitivity not only avoids the potential catastrophic consequences to the patient of a missed diagnosis but also means that in a patient with a negative test result a pheochromocytoma can be confidently excluded. By reliably excluding the presence of pheochromocytoma when the test is negative, no further tests are necessary. This alone would cut down on the current average of 3 to 4 tests performed on each patient to only 1 test per patient. At a cost of at least US\$100 per test (including costs at the clinic of sample collection as well as subsequent shipping and additional charges associated with insurance) and with up to 200,000 individuals screened for pheochromocytoma annually, this represents a potential savings to the United States health care system in unnecessary biochemical tests alone in excess of US\$50 million per year. A similar saving in the form of costs of unnecessary and more expensive CT, MRI and MIBG imaging studies could also be expected.

Of course, as with all biochemical tests, it can be expected that of the 200,000 tests of plasma metanephrines that would be required each year, at least $5 \%$ would be positive, with most of these representing false-positive test results. The incidence of false-positive test results can be expected to increase 
with each additional test that is carried out. Thus, reducing the number of initial diagnostic tests to a single test of plasma metanephrines can also be expected to lead to reduction in subsequent costs when one of the multiple tests is positive and further testing, including expensive imaging studies, is called for. Among the 10,000 positive test results that might be expected for a single test of plasma metanephrines (i.e., 5\% of 200,000 tests), about $10 \%$ can be expected to represent true-positive test results. Among these, about $70 \%$ can be expected to have plasma levels of metanephrines so high that the test results could only reflect a pheochromocytoma (i.e., as opposed to sympathoadrenomedullary activation). In these pa- tients, the clinician can immediately concentrate on the task of locating the tumor without the need for further biochemical tests. Because other tests do not show the same magnitude of increase associated with a pheochromocytoma, these other tests do not reliably prove the presence of the tumor in the same high proportion of patients as tests of plasma metanephrines. This can be expected to result in further cost reductions.

With the above considerations in mind it can be appreciated that tests of plasma metanephrines would not only improve the chances of survival for the patient with the tumor, but would also lead to considerable savings in costs for any health care system where the test is appropriately implemented.

\section{References}

1. Goldstein DS (1995). Stress, Catecholamines, and Cardiovascular Disease. Oxford University Press, New York.

2. Sinclair D, Shenkin A \& Lorimer AR (1991). Normal catecholamine production in a patient with a paroxysmally secreting phaeochromocytoma. Annals of Clinical Biochemistry, 28: 417-419.

3. Stewart MF, Reed P, Weinkove C, Moriarty KJ \& Ralston AJ (1993). Biochemical diagnosis of phaeochromocytoma: two instructive case reports. J ournal of Clinical Pathology, 46: 280-282.

4. Bravo EL (1994). Evolving concepts in the pathophysiology, diagnosis, and treatment of pheochromocytoma. Endocrine Reviews, 15: 356-368.

5. Lenders J W, Keiser HR, Goldstein DS, Willemsen JJ, Friberg $P$, J acobs MC, Kloppenborg PW, Thien T \& Eisenhofer G (1995). Plasma metanephrines in the diagnosis of pheochromocytoma. Annals of Internal Medicine, 123: 101-109.

6. Shawar L \& Svec F (1996). Pheochromocytoma with elevated metanephrines as the only biochemical finding. J ournal of the Louisiana State Medical Society, 148: 535-538.

7. Eisenhofer $G$, Lenders JWM, Linehan WM, Walther MM, Goldstein DS \& Keiser HR (1999). Plasma normetanephrine and metanephrine for detecting pheochromocytoma in von Hippel-Lindau disease and multiple endocrine neoplasia type 2. New England J ournal of Medicine, 340: 1872-1879.

8. Eisenhofer G, Goldstein DS \& Kopin IJ (1989). Plasma dihydroxyphenylglycol for estimation of noradrenaline neuronal reuptake in the sympathetic nervous system in vivo. Clinical Science, 76: 171-182.

9. Eisenhofer G (1994). Plasma normetanephrine for examination of extraneuronal uptake and metabolism of noradrenaline in rats. Naunyn-Schmiedeberg's Archives of Pharmacology, 349: 259-269.

10. Eisenhofer $G$, Friberg $P$, Rundqvist $B$, Quyyumi AA, Lambert G, Kaye DM , Kopin IJ , Goldstein DS \& Esler MD (1996). Cardiac sympathetic nerve function in congestive heart failure. Circulation, 93: 16671676.

11. Graefe KH \& Henseling M (1983). Neuronal and extraneuronal uptake and metabolism of catecholamines. General Pharmacology, 14: 27-33.

12. Eisenhofer G, Goldstein DS, Ropchak TG, Nguyen HQ, Keiser HR \& Kopin IJ (1988). Source and physiological significance of plasma 3,4-dihydroxyphenylglycol and 3methoxy-4-hydroxyphenylglycol. J oumal of the Autonomic Nervous System, 24: 114.

13. Goldstein DS, Eisenhofer G, Stull R, Folio CJ , Keiser HR \& Kopin IJ (1988). Plasma dihydroxyphenylglycol and the intraneu- ronal disposition of norepinephrine in humans. J ournal of Clinical Investigation, 81: 213-220.

14. Eisenhofer G, Pecorella W, Pacak K, Hooper D, Kopin IJ \& Goldstein DS (1994). The neuronal and extraneuronal origins of plasma 3-methoxy-4-hydroxyphenylglycol in rats. J ournal of the Autonomic Nervous System, 50: 93-107.

15. Eisenhofer G, Smolich JJ \& Esler MD (1992). Disposition of endogenous adrenaline compared to noradrenaline released by cardiac sympathetic nerves in the anaesthetized dog. Naunyn-Schmiedeberg's Archives of Pharmacology, 345: 160-171.

16. Brown M (1984). Simultaneous assay of noradrenaline and its deaminated metabolite, dihydroxyphenylglycol, in plasma: a simplified approach to the exclusion of pheochromocytoma in patients with borderline elevation of plasma noradrenaline concentration. European J ournal of Clinical Investigation, 14: 67-72.

17. Duncan MW, Compton P, Lazarus $L \&$ Smythe GA (1988). Measurement of norepinephrine and 3,4-dihydroxyphenylglycol in urine and plasma for the diagnosis of pheochromocytoma. New England J ournal of Medicine, 319: 136-142.

18. Atuk NO, Hanks J B, Weltman J, Bogdonoff DL, Boyd DG \& Vance ML (1994). Circulating dihydroxyphenylglycol and norepinephrine concentrations during 
sympathetic nervous system activation in patients with pheochromocytoma. J ournal of Clinical Endocrinology and Metabolism, 79: 1609-1614.

19. Nakada T, Sasagawa I, Kubota $Y$, Suzuki H, Ishigooka M \& Watanabe M (1996). Dihydroxyphenylglycol in pheochromocytoma: its diagnostic use for norepinephrine dominant tumor. J ournal of Urology, 155: 14-18.

20. Eisenhofer G, Aneman A, Hooper D, Rundqvist B \& Friberg P (1996). Mesenteric organ production, hepatic metabolism, and renal elimination of norepinephrine and its metabolites in humans. J ournal of Neurochemistry, 66: 1565-1573.

21. Mardh G, Luehr CA \& Vallee BL (1985). Human class I alcohol dehydrogenases catalyze the oxidation of glycols in the metabolism of norepinephrine. Proceedings of the National Academy of Sciences, USA, 82: 4979-4982.

22. Mardh G, Dingley AL, Auld DS \& Vallee BL (1986). Human class II (pi) alcohol dehydrogenase has a redox-specific function in norepinephrine metabolism. Proceedings of the National Academy of Sciences, USA, 83: 8908-8912.

23. Peaston RT \& Lai LC (1993). Biochemical detection of phaechromocytoma: Should we still be measuring urinary HMMA? J ournal of Clinical Pathology, 46: 734-737.

24. Neumann HP, Berger DP, Sigmund G, Blum U, Schmidt D, Parmer RJ , Volk B \& Kirste G (1993). Pheochromocytomas, multiple endocrine neoplasia type 2, and von Hippel-Lindau disease. New England J ournal of Medicine, 329: 1531-1538.

25. Tormey WP \& FitzGerald RJ (1995). Phaeochromocytoma - a laboratory expe- rience. Irish J ournal of Medical Science, 164: 142-145.

26. Mornex R \& Peyrin L (1996). The biological diagnosis of pheochromocytoma. Bulletin et Memoires de l'Academie Royale de Medecine de Belgique, 151: 269-277.

27. Peaston RT, Lennard TW \& Lai LC (1996). Overnight excretion of urinary catecholamines and metabolites in the detection of pheochromocytoma. J ournal of Clinical Endocrinology and Metabolism, 81: 13781384.

28. Eisenhofer $G$, Rundqvist $B$, Aneman $A$, Friberg P, Dakak N, Kopin IJ , J acobs MC \& Lenders J W (1995). Regional release and removal of catecholamines and extraneuronal metabolism to metanephrines. J ournal of Clinical Endocrinology and Metabolism, 80: 3009-3017.

29. Eisenhofer G, Friberg P, Pacak K, Goldstein DS, Murphy DL, Tsigos C, Quyyumi AA, Brunner HG \& Lenders JW (1995). Plasma metadrenalines: do they provide useful information about sympatho-adrenal function and catecholamine metabolism? Clinical Science, 88: 533-542.

30. Eisenhofer $\mathrm{G}$, Keiser H, Friberg P, Mezey $E$, Huynh TT, Hiremagalur B, Ellingson T, Duddempudi S, Eijsbouts A \& Lenders JW (1998). Plasma metanephrines are markers of pheochromocytoma produced by catechol-O-methyltransferase within tumors. J ournal of Clinical Endocrinology and Metabolism, 83: 2175-2185.

31. Roth J A (1992). Membrane-bound catechol-O-methyltransferase: a reevaluation of its role in the O-methylation of the catecholamine neurotransmitters. Review of Physiology, Biochemistry and Pharmacology, 120: 1-29.
32. Eisenhofer G \& Finberg J P (1994). Different metabolism of norepinephrine and epinephrine by catechol-O-methyltransferase and monoamine oxidase in rats. J ournal of Pharmacology and Experimental Therapeutics, 268: 1242-1251.

33. Lenders J WM , Eisenhofer G, Abeling NG, Berger W, Murphy DL, Konings $\mathrm{CH}_{\text {, }}$ Wagemakers LM, Kopin IJ , Karoum F, van Gennip AH \& Brunner HG (1996). Specific genetic deficiencies of the $A$ and $B$ isozymes of monoamine oxidase are characterized by distinct neurochemical and clinical phenotypes. J ournal of Clinical Investigation, 97: 1010-1019.

34. Box JC, Braithwaite MD, Duncan $T \& \&$ Lucas G (1997). Pheochromocytoma, chronic renal insufficiency, and hemodialysis: a combination leading to a diagnostic and therapeutic dilemma. American Surgeon, 63: 314-316.

35. Peyrin L, Cottet-Emard JM, Pagliari R, Cottet-Emard RM, Badet C \& Mornex R (1994). Plasma methoxyamines assay: a practical advance for the diagnosis of pheochromocytoma. Pathologie Biologie, 42: 847-854.

36. Karsdorp N, Elderson A, Wittebol-Post D, Hene RJ , Vos J, Feldberg MA, van Gils $A P$, J ansen-Schillhorn van Veen J $M$, Vroom TM, Hoppener JW \& Lips CJ M (1994). Von Hippel-Lindau disease: new strategies in early detection and treatment. American J ournal of Medicine, 97: 158-168.

37. Aprill BS, Drake AJ , Lasseter DH \& Shakir KM (1994). Silent adrenal nodules in von Hippel-Lindau disease suggest pheochromocytoma. Annals of Internal Medicine, 120: 485-487. 